\title{
The Development of Transactional Methodology in Social Sciences Based on the Grounded Theory Model
}

\author{
Rauf Rahimi \\ Department of Political Sciences, Faculty of Administrative Sciences and Economic, \\ University of Isfahan, Isfahan, I. R. Iran \\ rahimirauf.phd@gmail.com \\ Hossein Harsij \\ Department of Political Sciences, Faculty of Administrative Sciences and Economic, \\ University of Isfahan, Isfahan, I. R. Iran \\ harsij@gmail.com \\ Abbas Hatami \\ Department of Political Sciences, Faculty of Administrative Sciences and Economic, \\ University of Isfahan, Isfahan, I. R. Iran \\ D.A.Hatami@gmail.com
}

\section{Doi:10.5901/mjss.2014.v5n23p2296}

\section{Abstract}

The purpose of present study is to represent how the transactional methodology in social sciences is developed by using qualitative approach of grounded theory, setting up focus group workshops and conducting semi-structured interviews with some Iranian professors in the methodology of social sciences. This study used the qualitative approach of the grounded theory. In 2014, focus group workshops were set up, and semi-structured interviews were conducted with the professors of methodology in several groups. It is necessary to use qualitative methods in this research due to the recognition of grounded factors. Findings of this study on the basis of data analysis suggests that causal and structural conditions, confusion in choosing the type of methodologies, and imperfection of each methodology in social sciences lead to the different combination of qualitative and quantitative methods, and conducting transaction among the existing methodologies that is affected by the intervening conditions like mutual impacts of methodology and subject on each other as well as considering everything in its own context resulting in the transactional methodology. To conclude, developing transactional methodology paves the way for producing theories, conducting research to solve problems, fulfilling the local and global needs, and developing human knowledge. Finally, the transactional methodology is proposed as an alternative methodology to study a social phenomenon.

Keywords: Grounded theory, Social sciences, Transactional methodology, Qualitative and quantitative methods.

\section{Introduction}

Methodology is the way of thinking about social reality (Glaser and Strauss, 2009). However; research method is a set of procedures and techniques that is used for data collection and data analysis. What matters is that methodology helps us understand the true nature of social events. Human beings cannot reach the absolute truth. Yet, research would hopefully guide human on how to understand the mechanism of the universe including social world.

This paper is going to find an alternative methodology by utilizing grounded theory (GT). It means that a new theory or methodology can be derived directly from the data which are systematically collected and analyzed in the process of research. In this approach, data collection, data analysis and the final theory are closely related to each other. It starts from reality in such a way that the researcher lets the theory come out of the data collected. From the data collected, the key points are marked with a series of codes, namely open coding, axial coding, and selective coding, which are extracted from the text. The codes are grouped into similar concepts in order to make the data more workable. Data analysis is begun by open coding where we work on small parts of a text made out of words, phrases, and sentences. Following open coding, there comes axial coding whereby data are put back together in new ways by making connections between one category and its subcategories. Categories created by this process are then labeled. Finally, 
there selective coding happens with taking notes and establishing categories. This study, therefore, aims to investigate how transactional methodology is created in social sciences based on grounded theory. According to the abovementioned points, the following questions are to be answered:

1- What are the weaknesses of existing methodologies?

2- How grounded theory can help us to develop an alternative methodology?

To answer these questions, this paper is divided into two main parts: in the first part, the methodology is descriptively explained, and in the second part different types of coding are carried out, as well as data obtained from interview are analyzed on the basis of GT so that by description, conceptualization and modeling, it is specified how transactional methodology is created in social sciences.

\section{Methedology}

The evaluation of credibility in a qualitative research, by comparing concepts and determining its relationship with data, is carried out to determine whether theories are consistent under accurate scrutiny. The GT does it in the same fashion. Comparison, questioning, and sampling are the essential characteristics of the GT which make it incomparable to other models. The grounded theory method was developed by two sociologists, Barney Glaser and Anselm Strauss (Glaser, 1978, 1992; Glaser\& Strauss, 1967; Strauss, 1987). The stages of a research based on GT are description, conceptual organization, and theorization. By description, we mean the use of words or phrases to transfer the mental image of an event, scenery, excitement, or feeling, or explanation from the descriptor's perspective. Conceptual organization is to organize data according to certain features.

And finally, a theory is a set of concepts connected by sentences expressing the relationship, and used for investigating and anticipating phenomena.. To this end, researchers detect and derive whatever matters to them, and describe it according to its characteristics (Strauss and Corbin, 1995, Perry1998).

In GT, by theory we mean a set of developed categories systematically connected by sentences expressing the relationship to set the theoretical framework that explain social, psychological, educational, medical phenomena and others of their ilk. Sentences expressing the relationship make us understand that by who, what, when, where, why, how, and with what consequences a phenomenon has happened. Once concepts within a framework are associated with sentences expressing the relationship, the findings of the research surpass the stage of conceptual organization, and change into theory. In the GT, data collection, and data analysis is carried out alternately. Data analysis starts with the first interview and observation, leading to the subsequent interview and observation, and these in turn persist through subsequent analysis, interview, and field work (Berg and Lune, 2004).

Hence there remains a perpetual transaction between the researcher and the research process. Objectivity is necessary for making accurate and unbiased interpretation on events. On the other hand, sensitivity is required to understand different nuances of meaning, and discovering the relationship among concepts. Both objectivity and sensitivity is essential for discovering. The essence of discovery is establishing a strong relationship between what was previously known for us and what has been unknown until now' (Strauss, \& Corbin, 1987). The first technique is comparative thinking. Comparison makes the analyzer study data at dimension level. The rationale for making comparison is to stimulate the thought to detect characteristics, and their dimensions. Another technique for moving away from data and subjectivity is to employ different perspectives on a particular event. Other methods are done through interview, observation, and written reports.

Micro-analysis is necessary from the beginning of research project to achieve primary categories with their characteristics and dimensions. Analysis is not a static or structured process, but it is mostly a creative one where the analyzer moves among different kinds of coding, and freely employs analytical techniques to counter analytical works. Microanalysis consists of open coding and axial coding, employing different techniques. Posing question is an analytical technique implemented to pave the way for doing research as well as theoretical direct sampling.. Every researcher bears in mind to know how to put questions that make the research proceed toward the result. Our chief procedure in this methodology is comparison and questioning. These should be established continually during the analysis. The main point in the analysis is that not to suppose our situations, sayings, and perspectives as certain once they are in sympathy with those of respondents (Strauss, \& Corbin, 1990, Chrmaz,2011).

Sampling is a complex stage in a qualitative research. Participants in this kind of research is called informant. They are selected based on the information or experience they have in terms of the subject of research. This sampling is also known as theoretical sampling (Strauss, \& Corbin, 1987). In the GT, almost 50 interviews or observations are said enough for the study to be conducted (Sandeloswski, 1995). In qualitative research, saturation is closely related to the sample size, and reduplication shows the sufficiency of the sample size. In this study, after posing questions and setting 
the framework, and employing the properties of field studies, data collection is done through conducting semi-structured interviews with methodologists. The number of participants depends on the research question. In this study, snowball method is used for selecting sample. First, we visited one of the chief informants, a philosophy professor at human science and cultural studies research center, and after attending interview, he introduced us to his colleague. This process, introducing other informants successively, continued to saturation. From 2013 to 2014, there conducted some interviews among methodologists in universities and research centers of Tehran, Isfahan, and Qom through successive introduction. Reaching theoretical saturation, we summed the number of interviewees and understood that they exceeded 100. Sampling in this study was conducted based on the participants' consent.

\section{Open and Axial Coding: The Weaknesses of Existing Methodologies}

Interviewer: What are the weaknesses of existing methodologies, namely, positivist, Interpretive and critical? How grounded theory can help us to develop an alternative methodology in social sciences?

Opinions of interviewees until theoretical saturation: plurality of methodologies has multiplied the necessity of determining the way of using them in social sciences [plurality of methodologies and uncertainty in selecting one of them]. Existing methodologies are imperfect, and they are not sufficient to answer research questions [imperfection of each of existing methodologies in social sciences]. One can develop transactional methodology by overcoming the weaknesses of existing methodologies and conducting a transaction among existing methodologies [conducting a transaction among existing methodologies]. Every habitat has its own horizon. Therefore, these horizons should be taken into account [considering everything in its own context]. If the horizons of positivist, critical, and interpretive methodologies are closed, these can make a new alternative and approach. In this way, they can also cover the weaknesses of each other [transactional methodology as an alternative methodology]. Subject has an impact on methodology in such a way that these methodologies are employed by the researcher in terms of the subject [mutual impacts of methodology and subject on each other]. Transactional methodologies could be developed by combining qualitative and quantitative approaches in a different way in social sciences [different combination of qualitative and quantitative approaches]. Transactional methodology can also have some benefits such as: solution of local and global problems, theorization and facilitation in adopting appropriate methodology to do the research in local and global level. Transactional methodology creates a balance between different subjects of social sciences and its methodologies [transactional methodology fulfilling the needs of research].

\section{Analytical Notes: The Weaknesses of Existing Methodologies to Produce an Alternative Methodology as the Structure}

The first thing that comes to our mind in this paragraph is the concept of "transactional methodology". Now what does each of these concepts mean? Transactional means exchange, and methodology is how to think about phenomena, and events. I think it would be better to clarify the meaning of transactional methodology by comparing and contrasting it with other methodologies in social sciences. Transactional methodology is the one developed through bringing about critical and interpretive methodologies, making their horizons close to each other, and employing positivist methodology.

Therefore, creating a transaction in order to exchange the characteristics of critical, interpretive, and positivist methodologies can lead to developing transactional methodology in social sciences. What makes the transactional methodology important is that none of critical, interpretive, and positivist methodologies cannot implement inclusiveness in studying different subjects of social sciences, because while each of these methodologies claims that they are complete and comprehensive, it is not so. Only transaction between these methodologies can develop a complete and comprehensive methodology. In other words, in this study, critical, interpretive, and positivist methodologies are subordinate to transactional methodology. Thus, it is necessary to set a transaction among existing methodologies to have them adapted.

Considering neutrality of science, sensitivity to social conditions, possibility of using qualitative and quantitative approach, explaining social structures, connecting theory with action, regarding objectivity, subjectivity, and their relationship are some strong points of critical methodology. However, radicalism, ignorance of positive aspects of tradition, changing science into ideology, inflexibility, ignorance of criticisms, and having radical pessimism about the current situation and trying to reform them are some disadvantages of critical methodology that make it open to criticisms. Critical methodology can strengthen its weak points through conducting transaction in interpretive methodology. Carrying out a transaction between interpretive and positivist methodologies, it could derive conservative features of the mentioned methodologies in order to adjust its radical features. On the other hand, critical and positivist methodologies can pay 
attention to common knowledge like interpretive methodology in order to adjust their feature simply highlighting the expert knowledge. By doing so, critical and positivist methodologies can be less abstract being compatible with social realities, because common knowledge mostly involves these realities. They can also adjust their pessimistic and humiliating views over common knowledge through adopting and paying attention to it as a feature of interpretive methodology. The transaction between them cause the three methodologies pay attention to both common knowledge as well as expert knowledge, and propose more valid theories in social sciences. Interpretive methodology has set some goals for science.

The goal of science is the salvation of humans which is incompatible with the goal of science seeking reality. On the other hand, interpretive methodology considers the goal of science understanding the meanings of what human actors verbalize. Yet, actors sometimes cannot verbalize the meaning of their behavior due to the lack of complete knowledge, or some unintentional actions. Moreover, the behavior of some people is influenced by structures, so they don't know what the root of it is; however, they show either intentional behavior, or unintentional one. They might not display it because of some political, personal, or social reasons. Critical and interpretive methodologies do not consider the ultimate goal of science, while positivist methodology says that the ultimate goal of science is achieving the truth. Thus, interpretive and critical methodologies can derive these characteristics from the positivist methodology, and set aside their deviant objectives. So the simple emphasis of the interpretive methodology on common knowledge is not enough for understanding the behavior of the actors. On the other hand, the subject of social sciences is not simply related to the behavior of people--social structures, values, and traditions are included. Positivist methodology studies structures and observable behavior, and ignores the motivations and meanings of that behavior, overlooks the will of men, considering them subordinate to the community structures. On the other hand, it is critical methodology that is paying attention to the infrastructures and values. While interpretive methodology mostly pays attention to the will of man, the meaning of the behavior, and intention and motivation of people, it ignores the observable behavior and structures. It can be seen that despite structures, agents and values are subjects of social sciences; none of the above methodologies studies them comprehensively because each simply investigates some parts of these subjects. Hence, conducting transaction between these methodologies causes these subjects to be studied well under transactional methodology, so multilateralism will be achieved. Emphasizing on relative understanding and the fact that the truth is what one verbalizes has resulted in the formation of idealism and relativism in the interpretive methodology.

This methodology also says that the truth is unattainable because theorists believe that in the new era simulation and modeling due to the spread of information and communication devices, lying and the truth cannot be distinguished. Hence, it is difficult to determine what is real and what is fake, so it is believed that the truth is unattainable. But it is noteworthy that just as men make it difficult to distinguish lying from the truth through modeling and simulation, they can also discern the lying from the truth using advanced tools. Difficulty in achieving the truth does not mean it is unattainable. Being conservative, simple emphasis on description, simple emphasis on understanding, relativism, and subjectivism are some of the features of interpretive methodology which are considered the weaknesses of this methodology. However, social phenomena are the byproducts of community. Yet, social structures such as culture, laws, traditions, politics, economics, social institutions, customs, conventions, experiences, skills, and personality development, etc. cannot simply change after formation and internalization. These, too, provide agents with the opportunities and constraints. Therefore, there is also a stable social phenomenon, but still variable. Critical and interpretive methodologies put great emphasis on byproduct and variability, and do not consider stability and continuity of above-mentioned social structures.Conducting transaction between existing methodologies, critical and interpretive methodologies can adopt the stability of positivist methodology adapting their characteristics of radical change.

In this way stability along with variability occur in critical and interpretive methodologies. Moreover, the features of science are not value-free, it is right, but it should not be the central value in critical and interpretive methodologies. In this regard, this feature should be adapted especially in critical methodology, and besides putting emphasis on this principle, it should also be considered not to involve values. To harness the involvement of values, one can carry out a transaction between critical and interpretive methodologies on the one hand, and positivist methodology on the other. Remaining neutral in considering values is emphasized in positivist methodology, while the science mixed with values is highlighted in critical and interpretive methodologies; nonetheless, actually neither is science mixed with values nor neutral. It is necessary that these methodologies exchange some aspects of their features with each other in order to form the view that while science is not neutral, and values are involved in it, it is possible to harness their involvement. Therefore, transactional methodology in this study can fulfill this condition in itself for it assumes the mentioned methodologies as a subset. To participants the main concern of interpreters is to address the fundamental differences between the natural and social realities, as well as to select a proper method for investigating them.

But the methods of the natural sciences can be also appropriate for the social sciences being more or less modified. Therefore, the social sciences is neither completely independent of the natural sciences considering 
methodology, nor is it limited to the methodologies of natural sciences. Here, it is also required to conduct a transaction between the contrastive features of natural and social sciences, as well as interpretive and critical methodologies, and similarities of methodologies of natural and social sciences, and positivist methodology. Through the given intermediary features, the transactional methodology, which includes all positivist, interpretive, and critical methodologies based on our assumption, will come into existence. Putting emphasis simply on functionality leads to voluntarism, and eliminate the possibility of explanation, prediction and generalization; however, putting emphasis only on structures results in ignoring the will and motivation of human, and paying attention only on the structure and conversion of man into the object leads to achieving the generalizability and predictability in the positivist methodology; Both are flawed because men are placed between determinism and free will.

The interaction between structure and agent allows for the possibility of careful descriptions, explanations, and predictions; however, the domain of the possibility in social sciences, due to the role of human will, is not as extended as that of natural sciences. Developing interpretive, positivist and critical methodologies, while conducting a constant interaction between them, allows for the possibility of solving these problems and formation of transactional methodology. In this regard, a transaction can be carried out. Critical methodology pays attention to structures as well as agents, but studies neither completely; because of the fact that, it degrades common knowledge and does not take all of agents into account; however, it considers and informs those who are dominated. On the other hand, it criticizes social structures radically, and does not take their positive aspects into consideration and wants to change them. Positivist methodology can provide the interpretive methodology with its structural features, and interpretive methodology can provide positivist methodology with its voluntaristic features; therefore, these methodologies can have both features together, adapting their simple structural and voluntaristic features. This intermediary feature in the transactional methodology is realized as a set of methodologies. The interviewees believed that we should begin with hermeneutics, but should not at it, because we cannot explain.

We should start with dialog, but we should also explain. Social phenomena are of independent existence and can be judged but not in a relativistic manner. Putting strong emphasis on understanding, ignoring explanations, and putting great emphasis on differences and not similarities are some of the difficulties that interpretive methodology faces. Therefore it is necessary to naturalize these features to develop a more comprehensive methodology. Interpretive methodology can provide positivist methodology with its feature, i.e. understanding, while positivist methodology can also provide interpretive methodology with explanation during transaction, thereby they can obtain these features altogether.

This intermediary feature can also be realized in the transactional methodology. On the other hand, with regard to the sensitivity that critical methodology shows toward social change and improvement, it can pose structural obstacles in the path of development of different castes of society. Thus, conducting a transaction between the features of critical and interpretive methodologies, namely prescription and description, and using them are important in scientific research to develop these two methodologies because these two methodologies make it possible for us to understand structures and agents through interaction. To respondents of the study, explanations may not lead to predictions in social sciences, or predictions go wrong. Social scientists cannot make neutral predictions, but they try to achieve events by prediction. Hence we are not indifferent towards our predictions. We want our predictions come true. Therefore, the prediction does not necessarily rooted in explanations; predictions are made neutrally, as well. Yet, the most important task of a social scientist is the abstraction facts. Interpretive and critical methodologies can adopt generalization and prediction from the positivist methodology thereby they obtain a more stable rules for social sciences. Moreover, positivist methodology can take specific and unique features of interpretive and critical methodologies that disrupt the prediction and generalization to adapt its extreme predictions--following the unchangeable rules in the social sciences-- and make more cautious predictions. This feature, that is considering general and specific, can also be come true in the transactional methodology. Practical defending from critical theory is problematic, because it seems that this theory ignores the conformability; while it seems that even a critical theory should be tested against conformability/truth. Theories should not simply lead to change, but this change must be progressive. Whether such a change is progressive or not depends on the conformability of the explanations of the theory regarding the initial conditions; for example, only if the workers were oppressed in the world, the critical theory would come to save them. Whether they were oppressed or not is the problem related to the conformability of the explanation of the theory with the realities, ignored by critical methodology. In developing this methodology, it is necessary to fill this gap so that the results are valid based on this methodology.

Many actors , misunderstand the claims of critical theorists, resisting the claims of critical methodology. In their view, these claims are irrational. Critical theorists do not consider the resistance of actors to revise their theory, but rather take this as a reason to discuss and reflect on the previous views. It should be said that although a particular theory in the broader sense of time and place is determined, the major concepts and theories should be able to remain consistent across experiments. Therefore, to achieve credibility, we need to test. Emphasizing the testability of the research 
hypotheses, positivist methodology can test the qualitative theories provided by critical and interpretive methodologies in a quantitative fashion thereby determining the extent of the credibility of those theories. Therefore, conducting a transaction between positivist methodology, and critical as well as interpretive methodologies, one can either develop theories and test them, or implement a different combination of qualitative and quantitative approaches in social sciences. Therefore, based on participants' views, it is necessary to construct a new dualist relationship apart from the existing dualism in methodologies and concepts. By establishing a relationship between these dualities, many problems and differences over concepts and methodologies disappear. It seems necessary to consider the three inseparable elements to eliminate the existing dichotomy within existing methodologies.

\section{Analytical Note: Qualative And Quantitive Methods As A Strategy}

Another interesting concept is "a different combination of qualitative and quantitative approaches ". So what is it? The different combination of qualitative and quantitative approaches is used to gain the strength of each approach in order to eliminate problems. Both methods suffer from the common technical problems. One of the technical problems relates to the participants. In experimental and survey research, subjects are apprised of being studied which can cause consequences for research, because it makes the conditions of the study abnormal, and it cause the behaviors to be unrealistic. This has led researchers to collect data through non-reactive methods in field experiments where people do not know they have been studied.

One of these non-reactive methods is the collaborative observation where the researcher gradually becomes a member of the group, and other members forget that s/he is a researcher. Hybrid research methodologies seek to integrate and combine qualitative and quantitative data in different forms in order to have a better understanding of the research. The way qualitative and quantitative data are combined in hybrid research is based on the strategies associated with the approach of this research. There may be a sequential process where we start using qualitative approach having exploratory purposes, then continuing with quantitative approach and a wide sample in order to generalize results to the population. Here the results can be generalized. In contrast, a study may start a quantitative approach where the hypotheses are tested, and then continue with a qualitative approach in order to discover information about individuals or limited samples of the study.

Another strategy in different combination of qualitative and quantitative approaches can be using them simultaneously. Here, the researcher wants to collect data through two methods simultaneously. Then for the analysis, the qualitative and quantitative data are integrated. Another strategy is the conversion process. Therefore, the researcher follows the research project with a theoretical framework including qualitative and quantitative data where sequential or simultaneous process can be implemented. Different combination of approaches is used as a process model in this paper, based on the research subject and researcher's preference. Reading between the lines, it is concluded that data collection and data analysis can be done during all phases of the study, using both modes, with the different combination of the two. One can continually change the approaches where quantitative and qualitative data have a circular effect on each other. Each has advantages in its turn, and is not superior over the other. Moving through the approaches is necessary for the study. The quantitative and qualitative researches have specific roles in academic research. The problem is not which approach we can use, but is how to apply both to strengthen the research. Most researchers emphasize that we should use qualitative and quantitative approaches complementally. However, our respondents highlight the actual interaction between both quantitative and qualitative approaches during the research. Qualitative work should direct quantitative one, while quantitative work provide qualitative work with feedback. This process should be circular everywhere, yet evolving. Plurality in research methods can provide valuable results. One example is that we firstly use semi-structured interviews to obtain the initial model, then accept or reject it by quantitative methods. Hence, quantitative methods alone cannot answer. Models are derived through qualitative methods using interviews and library studies. The most important tool in this process is conducting interview. Once the researcher achieved the theoretical saturation, she/he proposes the initial model, and then rejects or accepts it by using quantitative methods.

\section{Axial Coding and Alternative Methdology}

These interviewees tell us in the form of a text what the transactional methodology in social sciences is, how it is created and why it is necessary. However, when we analyze the data, we translate the text into the following concepts that can be substituted for the words of the interviewees:

- plurality of methodologies and confusion in choosing the type of methodology; 
- establishing interaction between existing methodologies;

- considering everything in its own context;

- alternative methodology of transactional methodology;

- mutual impacts of methodology and subject on each other;

- different combination of quantitative and qualitative methodology;

- transactional methodology fulfilling the needs of research; and

- Imperfection of each methodology in social sciences.

Using these concepts which are sub-category of the main category of the transactional methodology, we illustrate the transactional methods in the social sciences. We have demarcated all of these codes in terms of their dimensions. So we have: "plurality" of methodologies and "confusion" in choosing the type of methodology; considering everything "in its own context"; alternative methodology of "transactional methodology"; mutual impacts of methodology and subject on each other; "bringing closer" the horizons of existing methodologies; "different combination" of quantitative and qualitative methodology; transactional methodology "fulfilling the needs of research"; and "imperfection" of each methodology in social sciences. So when we analyze the data, there are actually two levels of explanation. One is the level of verbal analysis which is done in open coding, and the other is the level of conceptualization analysis carried out in axial coding. "Transactional methodology in the social sciences" is what our respondents consider a possible methodology.

Our interpretation and definition of the phenomenon (transactional methodology) or what is going on in this situation, is that these structural conditions will be created by the following strategy or interactions: bringing closer the horizons of existing methodologies, establishing an interaction between existing methodologies and different combination of qualitative and quantitative methods. In the eyes of our interviewees, these are just some of the categories which can be used for developing the transactional methodology. Hence, conducting a transaction between the existing methodologies, it is necessary for us to know more features and dimensions of the methodology in order to develop them. This is our interpretation of the event.

\section{The Theoretical Model of Trnsactional Methodology in Social Sciences}

In the data, these categories were selected as sub-categories of the main category, and the theoretical model was developed. The causal conditions arose from this information which led to the formation of specific experiences in relation to the interaction among existing methodologies in social sciences. These causal conditions include: 1 . Plurality of methodologies, 2. confusion in choosing the type of methodology, and 3. imperfection of each methodology in social sciences. The Strategy or actions / interactions used to create the structural conditions are: "establishing an interaction between existing methodologies and different combination of qualitative and quantitative methods." These strategies were influenced by the grounded theory. The grounded signs include scope, frequency, intensity, and time. Based on the frequency, sub-categories are formed as the features of the main category, grounded signs, and situation. A comprehensive alternative methodology-- lacking the imperfections of the simple quantitative or qualitative methods, as well as the existing methodologies--has been introduced since the beginning of the formation of the hybrid research methodology. The scope the alternative methodology ranges from the humanities and social sciences to the empirical science and mathematics. The alternative methodology is a process that is constantly repeated and its intensity changes over time in scientific circles. It is not clear when the hybrid research methodology was introduced as an alternative methodology. This has fluctuated from the last decades of the 20th century up to now.

Due to the imperfections existing in the use of either qualitative or quantitative methodologies, pragmatic approach has emerged. The category of alternative methodology in the social sciences differs in terms of frequency, intensity, scope, and duration. Therefore, we are to propose a particular type the alternative methodology, namely transactional methodology to the scientific community, we recommend. In addition to the ground and situation, there have been intervening conditions. These conditions influence the interaction between the existing methodologies, and different combination of qualitative and quantitative methods. Based on the functions of the categories in coding such as "mutual impacts of methodology and subject on each other, and considering everything in its own context", intervening conditions are identified as mentioned. The outcomes of action / interaction dealing with the problematic structural conditions in the social sciences lead to the formation of transactional methodology. New actions / interactions will happen after the formation of transactional methodology, and they may have numerous outcomes. Figure 1 indicates the characteristics of theoretical model: 


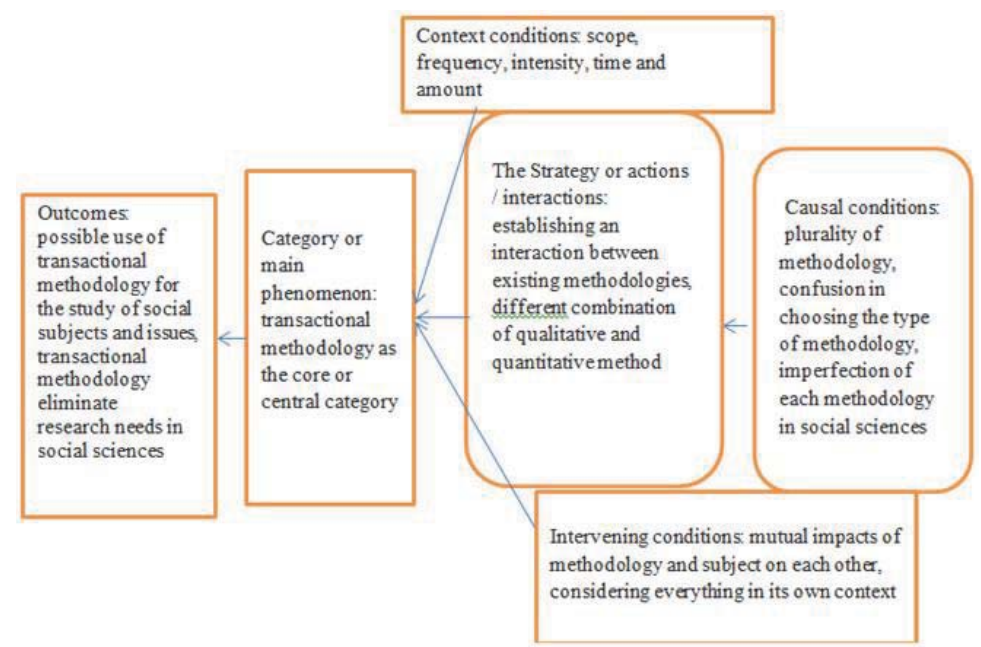

\section{Conclusion}

Everyday life is the context of the formation of social phenomena such methodology which is the key to the understanding of social relations. Although the methodology is the way to think about the human and social relations, its structure is not constant while created by various resources due to different social contexts and situations. That's why one of the most important issues in social sciences concerning the field of research methodology is how to use qualitative and quantitative methods in scientific research. Knowing the functions of qualitative and quantitative methods in social sciences research is one of the basic topics related to how to combine qualitative and quantitative methods to create a transactional methodology in the social sciences. Quantitative methods were dominant in the era of modernism, while qualitative methods have emerged in the era of postmodernism. Postmodern theorists have tried to challenge the unquestioned dominance of quantitative methods. Finally, the failure of both methods leads to the emergence of the hybrid research methodology in the second half of the twentieth century. However, in this study we revealed that no theoretical consensus exists over how we combine of the qualitative and quantitative methodologies. This study proposed the different combination of quantitative and qualitative methods as a process model for creating the transactional methodology. It was identified that each feature of these methodologies is subordinate to the transactional methodology as a proposed structural model in this paper.

Transactional methodology embraces some elements of objectivity and subjectivity, understanding, explanation and prediction, particularism and universalism, and stability and change in social phenomena, structure and agency, quantitative and qualitative methods, and similarity between methods of natural sciences and social sciences, unbiasedness of science and its combination to values, description, prescription, and explanation, theory and practice, etc. The relationship between the features is essential; therefore, we offer three essentialities instead of two where we don't simply claim that one of the features is essential, but accepting the essentiality of each feature, we believe that the relationship among the features is essential. If this is so, we will eliminate the dichotomy existing in the methodologies of social sciences. In this way, we will have a more comprehensive methodology for social sciences, because the social science is not concentrated in a particular subject in contrast to the views of the positivist, critical, and interpretive methodologies. However, it consists of structures, people, values, meanings and behavior and so on. Transactional methodology can conduct a transaction among the features of positivist, critical and interpretive methodologies through the interaction among its subset methodologies; therefore, its all-inclusiveness leads to better understanding of social phenomena.

\section{References}

Berg, B. L., \& Lune, H. (2004). Qualitative research methods for the social sciences (Vol. 5). Boston: Pearson.

Charmaz, K. (2011). Grounded theory methods in social justice research. Sage, Thousand Oaks, CA, 359-380.

Glaser, B. G., \& Strauss, A. L. (2009). The discovery of grounded theory: Strategies for qualitative research. Transaction Publishers.

Parry, K. W. (1998). Grounded theory and social process: A new direction for leadership research. The Leadership Quarterly, 9(1), 85105.

Sandeloswski, M. (1995). "Sample size in qualitative research. Research in nursing \& health", 18(2). :117-128.

Strauss, A. L. (1987). "Qualitative analysis for social scientists". Cambridge: Cambridge University Press. 
Strauss, A., \& Corbin, J. (1990). "Basics of qualitative research: Grounded theory procedures and techniques" Newbury park, CA: Sage. Strauss, A., \& Corbin, J. (1998)." Basics of qualitative research: Techniques and procedures for developing grounded theory" Thousand Oaks, CA: Sage Publications. 